should also be measured in all patients and an annual pure tone audiogram should be considered, especially for patients who receive frequent courses of tobramycin.

While tobramycin levels were slightly lower in the finger-prick samples, the very small mean difference found is only of limited clinical importance. We have previously observed this phenomenon when comparing immunosuppressant levels in finger-prick and venous samples using the same method for measurement [3]. Finger-prick blood (often referred to as capillary blood) is a mixture of arteriolar, venous and capillary blood, although thought to resemble arterial more than venous blood. In addition to blood, samples will also contain small volumes of interstitial and intercellular fluids, possibly accounting for the somewhat lower measured drug levels. Finger-prick blood collection can also be undertaken at home and this may allow the patient to post samples into the laboratory using the regular mail service. This simple technique could save the patient from having to make unnecessary trips to the hospital.

In conclusion, finger-pick sampling can provide a less invasive method than traditional venepuncture for the measurement of blood tobramycin levels in patients with CF.

Andrew Jones*,\#, Judith Beisty*, Diane McKenna*, Debbie Clough* $^{*}$ Kevin Webb*,\#, Julie Morris", ${ }^{\# \text { 厂 }}$ and Brian Keevil ${ }^{+}$ *Manchester Adult Cystic Fibrosis Centre, "Medical statistics, ${ }^{+}$Dept of Biochemistry, University Hospitals South Manchester NHS Foundation Trust, and \#Respiratory Research Group, Faculty of Medical and Human Sciences, The University of Manchester, Manchester Academic Science Health Centre, Manchester, UK.

Correspondence: A. Jones, Manchester Adult Cystic Fibrosis Centre, University Hospitals South Manchester NHS Trust,
Southmoor Road, Manchester, M23 9LT, UK. E-mail: andrew. jones@uhsm.nhs.uk

Statement of Interest: None declared.

\section{REFERENCES}

1 Antibiotic Treatment for Cystic Fibrosis. Report of the UK Cystic Fibrosis Trust Antibiotic Working Group. 3rd Edn. Cystic Fibrosis Trust, 2009.

2 Smyth AR. Minimizing the toxicity of aminoglycosides in cystic fibrosis. J R Soc Med 2010; 103: Suppl. 1, s3-s5.

3 Webb NJ, Roberts D, Preziosi R, et al. Fingerprick blood samples can be used to accurately measure tacrolimus levels by tandem mass spectrometry. Pediatr Transplant 2005; 9: 729-733.

4 Merton G, Jones K, Lee M, et al. Accuracy of cyclosporine measurements made in capillary blood samples obtained by skin puncture. Ther Drug Monit 2000; 22: 594-598.

5 Woods K, Douketis JD, Schnurr T, et al. Patient preferences for capillary $v s$ venous INR determination in an anticoagulation clinic: a randomized controlled trial. Thromb Res 2004; 114: 161-165.

6 Bland JM, Altam DG. Statistical methods for assessing agreement between two methods of clinical measurement. Lancet 1986: 1; 307-310.

7 Pitt T, Sparrow M, Warner M, et al. Survey of resistance of Pseudomonas aeruginosa from UK patients with cystic fibrosis to six commonly prescribed antimicrobial agents. Thorax 2003; 58: 794-796.

8 Mogayzel PJ, Pierce E, Mills J, et al. Accuracy of tobramycin levels obtained from central venous access devices in patients with cystic fibrosis is technique dependent. Pediatr Nurs 2008; 34, 6: 464-469.

9 Struthers SL, Nicolson T, Jones G, et al. Falsely elevated serum tobramycin levels in a patient receiving nebulised tobramycin. J Cyst Fibros 2002; 1: 146-147.

10 Madsen V, Lind A, Rasmussen $\mathrm{M}$, et al. Determination of tobramycin in saliva is not suitable for therapeutic drug monitoring of patients with cystic fibrosis. J Cyst Fibros 2004; 3: 249-251.

11 Mulrennan SA, Helm J, Thomas RB, et al. Aminoglycoside ototoxicity susceptibility in cystic fibrosis. Thorax 2009; 64: 271-272.

DOI: $10.1183 / 09031936.00176611$

\title{
Noninvasive ventilation in cystic fibrosis: the Italian physiotherapists' point of view
}

\section{To the Editors:}

A physiological rationale has been demonstrated for the use of noninvasive ventilation (NIV) in patients with cystic fibrosis (CF) [1]; however, there is little evidence [2] to support NIV as a routine clinical treatment in people with $\mathrm{CF}$, especially for those with advanced lung disease. Possible explanations include the lack of clearly validated criteria to propose NIV, controversies with regard to the optimal ventilatory modes and settings, scepticism with regard to long-term efficacy [3], poor acceptance by some patients and, above all, lack of familiarity with this technique among health workers. Instead, respiratory physiotherapists are often involved in the NIV management with different skills and tasks [4]. The aim of this study was to survey and evaluate the role and competencies of Italian physiotherapists involved in NIV management in CF centres and to understand the rationale of NIV as perceived by them. A semi-structured online questionnaire consisting of 31 closed and nine open-ended questions was sent to the Italian Group of Physiotherapists $(n=63)$ belonging to the Italian Society for the Study of Cystic Fibrosis, between March and April 2010. Respondents ( $n=42,67 \%)$ worked in 24 CF centres (18 centres and six clinics), which had a total of $4,064 \mathrm{CF}$ patients at the time of the study, $96(2.4 \%)$ of those were using NIV. NIV was the therapeutic choice used in $63 \%$ of those centres and 28 (93\%) out of 30 physiotherapists were involved 


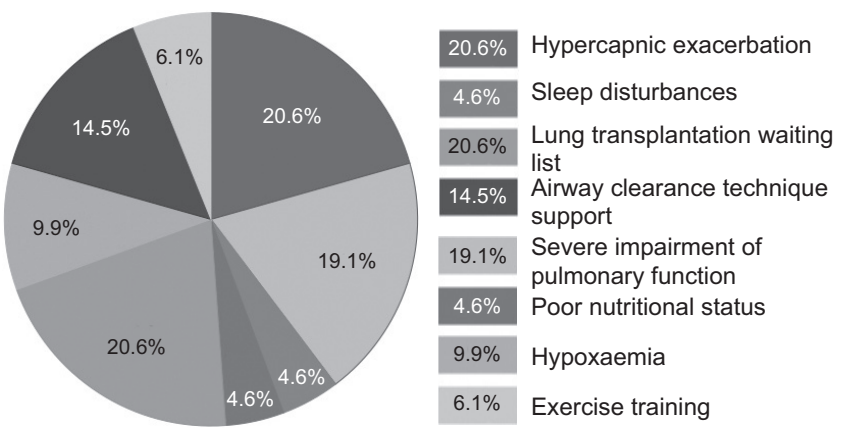

FIGURE 1. The main criteria for noninvasive ventilation initiation according to physiotherapists.

in its management. According to respondents, NIV was the first-line treatment to improve gas exchange (89\%) and was a valid tool as support to clearance techniques (68\%) and to exercise training (43\%). The main criteria to propose NIV, according to physiotherapists who use NIV $(n=28)$, were hypercapnic pulmonary exacerbation, awaiting lung transplantation, severe impairment of pulmonary function and difficulties experienced with clearing secretions (fig. 1). Almost all interviewees agreed that NIV was part of the "core competence" for physiotherapists who work in CF centres $(95 \%)$ and clinics $(85 \%)$. A detailed picture revealed that $71 \%$ $(n=20)$ of them chose ventilators, $96 \%$ were involved in the crucial choice of interfaces and $75 \%$ were in charge of ventilator settings. The majority (93\%) took care of patients' comfort and compliance enhancement and set frequent follow-ups to periodically review patients and, thus, improve their adherence. Although there are some limitations, this is the first Italian study that explores how physiotherapists are involved in the NIV management in CF centres and their expectations and beliefs about the benefits of NIV. Despite the absence of standard protocols and guidelines, Italian CF centres and clinics take into consideration the therapeutic use of NIV and physiotherapists are involved in its use. According to our results, the rationale of adopting NIV as a therapeutic choice in CF as perceived by physiotherapists is in line with what the literature has recently suggested [5]. Harmonising the core competencies of physiotherapists all over Italy and the NIV management of CF patients is, however, still urgently needed.

\section{Sergio Zuffo*, Simone Gambazza ${ }^{\#}$ and Alessandro Capra* *Rehabilitation Unit, and "Cystic Fibrosis, Azienda Ospedaliero Universitaria A. Meyer, Florence, Italy.}

Correspondence: S. Gambazza, Azienda Ospedaliero Universitaria Meyer, c/o Fibrosi Cistica, Viale Pieraccini 24, 50139, Florence, Italy. E-mail: simone_gambazza@hotmail.it

Statement of Interest: None declared.

\section{REFERENCES}

1 Fauroux B, Hart N, Lofaso F. Non invasive mechanical ventilation in cystic fibrosis: physiological effects and monitoring. Monaldi Arch Chest Dis 2002; 57: 268-272.

2 Moran F, Bradley JM, Piper AJ. Non-invasive ventilation for cystic fibrosis. Cochrane Database Syst Rev 2009; 1: CD002769.

3 Fauroux B, Le Roux E, Ravilly S, et al. Long-term noninvasive ventilation in patients with cystic fibrosis. Respiration 2008; 76: 168 174.

4 Moran FM, Bradley JM, Elborn JS, et al. Physiotherapy involvement in non-invasive ventilation hospital services: a British Isles survey. Int J Clin Pract 2005; 59: 453-456.

5 Fauroux B. Non invasive ventilation in cystic fibrosis. Expert Rev Resp Med 2010; 4: 39-46.

\section{Penicillium marneffei presenting as an obstructing endobronchial lesion in an immunocompetent host}

\section{To the Editors:}

Penicillium marneffei was first isolated in 1956 from the liver of the Vietnamese bamboo rat [1]. It is recognised as an emerging infection among HIV-infected patients in South East Asia, where it typically presents as cutaneous lesions, fever, lymphadenopathy and hepatosplenomegaly [2]. Indeed, in this population, it is now the third most common opportunistic infection [3]. Penicilliosis is a rare disease in immunocompetent hosts. It has been previously described in patients with comorbid conditions such as connective tissue diseases and haematological malignancies. Although inhalation of the conidia is thought to lead to disseminated infection, a primary pulmonary presentation is unusual. We describe a unique presentation of systemic $P$. marneffei infection in an immunocompetent host presenting with an endobronchial tumour-like lesion and postobstructive pneumonia.

A 45-yr-old, previously well male was referred to our hospital for further investigation of fevers, lymphadenopathy and pulmonary infiltrates. The patient described a 4-month history of intermittent drenching night sweats, cough and posterior cervical lymphadenopathy against a background of a recent bicycle tour of Laos and Vietnam. There was no significant family history, no significant occupational exposure and a trivial smoking history. The patient was initially investigated at a regional hospital with apparently normal chest and abdominal imaging and mild eosinophilia on a peripheral blood film. Excisional biopsy of an enlarged cervical lymph node demonstrated caseating granulomas with no evidence of 